\title{
China Regional Resource and Economic Coordinated Development Research
}

\author{
Liu yi ${ }^{1}$, Zhang Mingrui ${ }^{1}$, Liu Yinghe ${ }^{2}$ \\ ${ }^{1}$ College of Economics and Administration, Harbin University of Science and Technology,Harbin,China, 150080 \\ ${ }^{2}$ Freshman University of washington
}

\begin{abstract}
Eastern, mid and western China were taken as research objects, and the data from 2007-2016 and evaluating regional resource and economic development were selected in this paper. The result shows that the development level from domestic region fluctuates in small scope with an obvious regional difference during the study period. (Eastern region has the highest level of coordinated development followed by mid and western region.)
\end{abstract}

\section{Introduction}

So far, Chinese economic has entered in the new age, which lead to the new age of politics, culture, ecological civilization and construction. As more and more environmental problems appear, our key point is how to promote the balance between resource environment and economic development. The research on the coordinated development of resources, environment and economy began in1960s. Influenced by the determinism of Western Keynes's economic development, it advocates that economic development decides everything, which brings about a series of environmental pollution problems, and the most typical problem is the emergence of the eight major public hazards in the world. When people rethink the mode of economic development and consider how to promote the economic development of social welfare under the constraints of environmental resources, people gradually realize that high-speed economic growth will pay a heavy price to the environment. "The limits to Growth" by the Club of Rome took globe as research subject for the first time to research world population growth and economic development. In China, a large number of research results have emerged since 1990s in the empirical research on the coordination of regional resources and environment and economic development. Meng Zhaoyan (2012) selected 20 environmental and economic indicators that affected the coordinated development of the ecological environment and the economy in Hebei Province, with a relatively wide coverage and more applicable and evaluated coordinated development of the environment and economy in Hebei by establishing a coordination degree model.

\section{Index system and computing methods}

\subsection{Research method and model}

Resource environment is the base of economic development, which is the product of the resource system. The resource and environment system provides a large amount of resources to the economic system, as the raw material of the economic production process, and the produced products meet the needs of human beings. The two constitute the organic whole which is interconnected and mutually restricted. The material exchange, energy flow and information transfer are continuously carried out within the complex system.

To measure coordination of two subsystems in certain region, it is necessary to establish related model. The coordinated development of resources, environment and economy is described by the degree of coordination, that is, coordinated development degree. Better coordination development demonstrates better system development. According to plenty of foreign and domestic document, a coordinated development degree model is proposed:

$$
\begin{gathered}
C=\left[\frac{f(x) \cdot g(x)}{(f(x)+g(x))^{2}}\right]^{k} \\
\mathrm{P}=\alpha \mathrm{f}(\mathrm{x})+\beta \mathrm{g}(\mathrm{x}) \\
\mathrm{D}=\sqrt{\mathrm{C} \cdot \mathrm{P}}
\end{gathered}
$$

$\mathrm{f}(\mathrm{x})$ represents the comprehensive score of the economic subsystem, and $\mathrm{g}(\mathrm{x})$ represents the comprehensive score of the resource and environment subsystem. The comprehensive score is mainly used to improve the entropy method. The specific algorithm is not detailed in this paper, and can be seen in the references. $\alpha$ and $\beta$ are the weight of the subsystem, and usually takes $0.5 . \mathrm{k}$ is the adjustment coefficient, and the value of this paper is 2. $D \in[0,1]$ is the coordinated development of the system.

Four stages of value: 
$[0.0,0.3]$ low coordination

$[0.3,0.5]$ middle coordination

$[0.5,0.8]$ high coordination

$[0.8,1.0]$ extreme coordination

\subsection{Index selection and data source}

Eastern, middle and western part were mainly analysed in this article. The system consists of resource environment subsystem and economic subsystem, each containing 6 indicators.

The index of environmental system: $\mathrm{SO} 2$ discharge, wastewater discharge, coal consumption, forest coverage rate, garbage harmless treatment rate,coverage rate of green area.

The index of economic: average wage, per capita fiscal revenue, per capita GDP, tertiary sector index, per capita housing investment, GDP index. The data from 30 regions of China 2007-2016 years (Tibet because of lacking of information, so the data are excluded from the research object). All the data are derived from the China Statistical Yearbook and the China Energy Statistics Yearbook from 2007 to 2016.

\section{Level of regional coordinated development}

According to the selection of the above methods and models, the relevant data are selected to make an objective evaluation of the coordinated development level of resources, environment and economy in various regions of China from 2007 to 2016. In order to investigate the regional differences in the level of coordinated development in each region, this paper divides the three regions in the eastern, central and western regions, and the specific results are shown in Table 1.

Table 1. Coordinated development of resources, environment and economy in China

\begin{tabular}{|c|c|c|c|c|c|c|c|c|c|c|c|}
\hline \multirow{13}{*}{ Eastern } & Region & 2007 & 2008 & 2009 & 2010 & 2011 & 2012 & 2013 & 2014 & 2015 & 2016 \\
\hline & Beijing & 0.678 & 0.683 & 0.765 & 0.748 & 0.72 & 0.732 & 0.727 & 0.718 & 0.659 & 0.681 \\
\hline & Tianjin & 0.609 & 0.594 & 0.597 & 0.615 & 0.635 & 0.597 & 0.554 & 0.567 & 0.55 & 0.555 \\
\hline & Hebei & 0.331 & 0.383 & 0.433 & 0.435 & 0.45 & 0.379 & 0.331 & 0.446 & 0.437 & 0.376 \\
\hline & Liaoning & 0.548 & 0.594 & 0.617 & 0.624 & 0.646 & 0.65 & 0.641 & 0.566 & 0.464 & 0.359 \\
\hline & Shanghai & 0.508 & 0.501 & 0.54 & 0.541 & 0.457 & 0.524 & 0.525 & 0.531 & 0.494 & 0.473 \\
\hline & Jiangsu & 0.59 & 0.615 & 0.64 & 0.624 & 0.616 & 0.62 & 0.621 & 0.622 & 0.604 & 0.598 \\
\hline & Zhejiang & 0.663 & 0.655 & 0.683 & 0.669 & 0.655 & 0.665 & 0.674 & 0.678 & 0.678 & 0.667 \\
\hline & Fujian & 0.509 & 0.534 & 0.546 & 0.537 & 0.571 & 0.594 & 0.643 & 0.627 & 0.64 & 0.597 \\
\hline & Shandong & 0.492 & 0.549 & 0.542 & 0.532 & 0.539 & 0.532 & 0.548 & 0.566 & 0.547 & 0.532 \\
\hline & Guangdong & 0.604 & 0.591 & 0.608 & 0.602 & 0.583 & 0.57 & 0.57 & 0.593 & 0.613 & 0.614 \\
\hline & Hainan & 0.397 & 0.447 & 0.512 & 0.587 & 0.604 & 0.591 & 0.67 & 0.633 & 0.612 & 0.593 \\
\hline & Average & 0.539 & 0.559 & 0.589 & 0.592 & 0.589 & 0.587 & 0.591 & 0.595 & 0.573 & 0.55 \\
\hline \multirow{10}{*}{ Middle } & Shanxi & 0.439 & 0.403 & 0.365 & 0.435 & 0.448 & 0.462 & 0.388 & 0.3 & 0.338 & 0.274 \\
\hline & Neimenggu & 0.55 & 0.619 & 0.628 & 0.612 & 0.645 & 0.623 & 0.56 & 0.552 & 0.562 & 0.545 \\
\hline & Jilin & 0.445 & 0.532 & 0.493 & 0.44 & 0.486 & 0.512 & 0.405 & 0.354 & 0.397 & 0.402 \\
\hline & Heilongjiang & 0.282 & 0.392 & 0.362 & 0.363 & 0.438 & 0.406 & 0.338 & 0.34 & 0.324 & 0.243 \\
\hline & Anhui & 0.317 & 0.398 & 0.417 & 0.465 & 0.476 & 0.515 & 0.525 & 0.508 & 0.468 & 0.417 \\
\hline & Jiangxi & 0.174 & 0.301 & 0.273 & 0.309 & 0.318 & 0.322 & 0.35 & 0.437 & 0.413 & 0.371 \\
\hline & Henan & 0.356 & 0.377 & 0.378 & 0.354 & 0.401 & 0.354 & 0.355 & 0.435 & 0.401 & 0.347 \\
\hline & Hubei & 0.357 & 0.409 & 0.428 & 0.446 & 0.468 & 0.473 & 0.502 & 0.568 & 0.525 & 0.488 \\
\hline & Hunan & 0.357 & 0.455 & 0.366 & 0.382 & 0.381 & 0.437 & 0.448 & 0.515 & 0.434 & 0.373 \\
\hline & Average & 0.364 & 0.432 & 0.412 & 0.423 & 0.451 & 0.456 & 0.43 & 0.445 & 0.429 & 0.384 \\
\hline \multirow{11}{*}{ Western } & Guangxi & 0.33 & 0.354 & 0.418 & 0.373 & 0.329 & 0.319 & 0.398 & 0.325 & 0.362 & 0.323 \\
\hline & Chongqing & 0.45 & 0.505 & 0.555 & 0.58 & 0.596 & 0.63 & 0.651 & 0.629 & 0.604 & 0.558 \\
\hline & Sichuan & 0.33 & 0.363 & 0.459 & 0.444 & 0.469 & 0.5 & 0.469 & 0.493 & 0.444 & 0.416 \\
\hline & Guizhou & 0.313 & 0.34 & 0.31 & 0.235 & 0.414 & 0.458 & 0.554 & 0.52 & 0.482 & 0.412 \\
\hline & Yunnan & 0.19 & 0.265 & 0.364 & 0.259 & 0.358 & 0.413 & 0.555 & 0.319 & 0.38 & 0.36 \\
\hline & Shanxi & 0.443 & 0.559 & 0.557 & 0.512 & 0.552 & 0.605 & 0.595 & 0.562 & 0.511 & 0.453 \\
\hline & Gansu & 0.147 & 0.342 & 0.299 & 0.226 & 0.299 & 0.412 & 0.435 & 0.387 & 0.327 & 0.264 \\
\hline & Qinghai & 0.375 & 0.456 & 0.414 & 0.487 & 0.522 & 0.546 & 0.509 & 0.509 & 0.462 & 0.454 \\
\hline & Ningxia & 0.346 & 0.475 & 0.469 & 0.517 & 0.517 & 0.57 & 0.538 & 0.494 & 0.49 & 0.486 \\
\hline & Xinjiang & 0.358 & 0.372 & 0.322 & 0.38 & 0.524 & 0.561 & 0.568 & 0.573 & 0.535 & 0.427 \\
\hline & Average & 0.328 & 0.403 & 0.417 & 0.401 & 0.458 & 0.501 & 0.527 & 0.481 & 0.46 & 0.415 \\
\hline
\end{tabular}

According to the data above, we can tell that there was small fluctuation of resource environment and economic coordination in different regions. In 2016, most of eastern region are in high-level coordination, most of western and mid region are in mid-level coordination, and several regions are still in low-level coordination. Comparing year 2007 and 2016, Jiangxi has the most improvement of $113 \%$ in coordination, following by that of $89 \%$ of Yunnan province. Shandong, Anhui, Hubei, Chongqing, Guizhou, Qinghai, Ningxia also have obvious improvement. Some regions have slight drop.

According to the data above, we can also tell that the coordination development and economic development in 
our country have a positive correlation with regional difference: Regions that have better coordination are mostly the eastern regions that have better economic development. Regions that have less coordination are mostly western regions that have behindhand economic development. The line chart downwards shows the average coordination in eastern and western region, reflecting the difference among different regions.

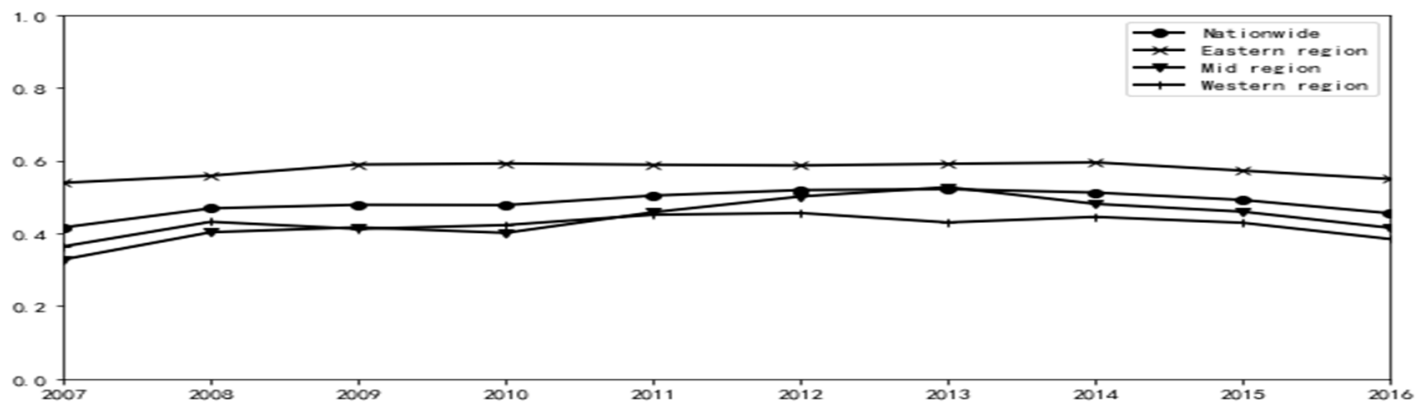

Figure 1. Mean trend chart of coordinated development degree in China

According to the graph above, eastern region in China has the highest-level coordination between environment and economy, which also obey the average level in the whole country. Western and mid region are lower than domestic average level. The reason for this is that the higher level of economic development in the eastern region provides a rich material basis for the coordinated development of the region. The level of social development, the level of science and technology and culture and the quality of the environment in the eastern region are far higher than that of the central and Western Regions. The second reason is the upgrading and transfer of the industrial structure. The heavy pollution and high energy consumption industries transferred from the east to the central and western regions, resulting in the deteriorating quality of the coordinated development of the environmental subsystems in the central and western regions and the slow rise of regional coordinated. The third reason is the pressure of economic growth. The central and western regions often regard economic development as the primary goal. Insufficient attention to environmental protection, social infrastructure construction and the improvement of people's quality of life result in a relatively low level of coordinated regional development.

\section{Conclusions and suggestion}

From the analysis above, there is a big difference of coordination in our country, so we need to improve the economic quality and promote interaction between regions to minimize the difference between regions. Economic development will have to face the challenge from resource basement, so the real coordination between economy and ecology must be realized. More efforts are needed in the following areas.

\subsection{Upgrading the industrial structure}

To realize the optimization and upgrading of industrial structure, the government need to provide industrial support for improving the comprehensive efficiency of regional coordinated development level. While maintaining the competitive advantage of the laborintensive industry in the international market, we should develop the high-tech industry and the new service industry from the policy guidance and the environment construction, and cultivate the new driving force for the economic growth. On the other hand, we should strengthen the constraints of the environmental governance, and apply the high technology. We should apply technological transformation to upgrade traditional industries. At the same time, all regions should improve the distribution and trading system of emission permits so that enterprises can bear the social cost of environmental pollution.

\subsection{Develop low carbon economic and adjust source consumption}

To improve the utilization of energy, firstly the government need to reinforce policy on low carbon combining with liable policy. Secondly, government need to guide enterprise to develop renewable energy such as wind, nuclear and solar power. Thirdly, introduce abroad low-carbon technology and put in more research to improve the utilization of the energy.

\subsection{Upgrade resource in the society}

As human capital and technology belong to scarce resources, they always flow to industries with higher profits, while the provision of public goods and environmental protection is nonprofit. Under the control of the market, the human capital and scientific and technological resources obtained by such economic activities are relatively small. So all regions should keep good personal training and develop advanced technology based on the needs of local economic development, keeping good personal training and the advanced technology, each region ought to have more cultural communication and make improved policy as well, which is really significant to western region. 


\section{Acknowledgment}

Heilongjiang Social Science Fund Project (number: 13B019)

Heilongjiang Social Science Federation Fund Project: (number: JD2016021)

\section{References}

1. Hongliang Liu, Fengzhong Cao, Yun $\mathrm{Xu}, \mathrm{Xu}$ Song[J]. China environment management, 2015, 7 (04):21-24.

2. Jingming Wei, Guang Cao, Man Xiong, $[\mathrm{J}]$. Heilongjiang resource economic district,2006(09): 45-48. China mining industry

3. Meadows, Donella H.,et al. The Limits to Growth: A Report for the Club of Rome[M]. Project on the Predicament of Mankind. Universe Books, New York, 1972.

4. Yuqian Zhang, Xiaofei Wang, Heilongjiang province economic and environment coordination policy[J].China forestry industry,2005(05):21$22+33$.

5. Jiutian Feng, Jianzhong Yin,resource-environmenteconomic development policy[J]. China population, 2005(03):135-139.

6. Zhaoyan Meng, Xiaoyang Yuan,Hebei environment and economic coordination[J].Shandong agricultural management ,2012,29(03):91-92.

7. Zhongbin [J].tropical geography, 1999(02):76-82.

8. Qi Wang, Fanghua Tang, Dongting lake ecologyeconomic-sociology[J].economiceograpphy,2015, 35 (12):161-167+202.

9. Rongtian Zhang, huafu Jiao[J]. Yangtze River resource and environment,2015,24(05):719-727.

10. Grant Allan,Nick Hanley,Peter McGregor,Kim Swales,Karen Turner. The impact of increased efficiency in the industrial use of energy: A computable general equilibrium analysis for the United Kingdom[J]. Energy Economics,2006,29(4).

11. XIONG Chuanhe,YANG Degang,HUO Jinwei, ZHAO Yannan.The relationship between energy consumption and economic growth and the development strategy of a low-carbon economy in Kazakhstan[J].Journal of Arid Land,2015,7(05):706-715.

12. Ying Song, Panyang Lake ecology resource[D].Jiangxi University of finance and economics, 2017.

13. Han Shengjuan,Zhu Jingping. Research on the Dynamic Relationship of the Energy-EconomyEnvironment (3E) System-Based on an Empirical Analysis of China[J]. Energy Procedia,2011,5.

14. $\mathrm{Na} \mathrm{Ta}$,Xiaoli Ning, Wuhai ecology and economic coordination $[\mathrm{J}]$. arid region resource and environment,2017,31(05):94-99.

15. C. Oliveira Henriques,C. Henggeler Antunes. Interactions of economic growth, energy consump- tion and the environment in the context of the crisisA study with uncertain data[J]. Energy,2012,48(1). 